\title{
hZip1 (hSLC39A1) regulates zinc homoeostasis in gut epithelial cells
}

\author{
Agnes A. Michalczyk • M. Leigh Ackland
}

Received: 7 August 2012/ Accepted: 18 January 2013/Published online: 2 February 2013

(C) Springer-Verlag Berlin Heidelberg 2013

\begin{abstract}
Zinc is an essential trace element required for enzyme catalysis, gene regulation and signal transduction. Zinc absorption takes place in the small intestine; however, the mechanisms by which cells accumulate zinc are not entirely clear. Zip1 (SLC39A1) is a predicted transmembrane protein that is postulated, but not conclusively proven to mediate zinc influx in gut cells. The aim of this study was to investigate a role for hZip1 in mediating zinc uptake in human enterocytes. Both hZip1 mRNA and protein were detected in human intestinal tissue. In nondifferentiated Caco-2 human gut cells, hZip1 was partially localised to the endoplasmic reticulum. In contrast, in differentiated Caco- 2 cells cultured in extracellular matrix, the hZip1 protein was located in proximity to the apical microvilli. Lack of surface antibody binding and internalisation indicated that hZip1 was not present on the plasma membrane. Functional studies to establish a role for hZip1 in cellular zinc accumulation were carried out using ${ }^{65} \mathrm{Zn}$. In Caco-2 cells harbouring an hZipl overexpression construct, cellular zinc accumulation was enhanced relative to the control. Conversely, Caco-2 cells with an hZipl siRNA construct showed reduced zinc accumulation. In summary, we show that the Caco-2 cell differentiation endorses targeting of hZip1 to a region near the apical domain. Given the absence of hZip1 at the apical plasma membrane, we propose that hZip1 may act as an intracellular sensor to regulate zinc homoeostasis in human gut cells.
\end{abstract}

A. A. Michalczyk · M. L. Ackland ( $\varangle)$

Centre for Cellular and Molecular Biology, School of Life and Environmental Sciences, Deakin University, 221 Burwood Highway, Burwood, Melbourne, VIC 3125, Australia

e-mail: leigha@deakin.edu.au
Keywords Zinc homoeostasis - Zinc transporter . hZip1 (SLC39A1) · Human gut

\section{Introduction}

The gut plays a key role in $\mathrm{Zn}$ homoeostasis through the absorption of exogenous $\mathrm{Zn}$ and the excretion of both endogenous and exogenous $\mathrm{Zn}$. The mechanisms by which the gut maintains tight homoeostatic control of $\mathrm{Zn}$ are not clear, but may involve members of two major families of putative $\mathrm{Zn}$ transporters, the ZnT (SLC30) and the Zip (SLC39) families. The hZip4 $\mathrm{Zn}$ transporter is implicated in $\mathrm{Zn}$ uptake in the human enterocyte as its gene is mutated in the disorder acrodermatitis enteropathica, a condition where $\mathrm{Zn}$ absorption in the intestine is impaired, leading to depletion of body $\mathrm{Zn}$ stores and characteristic acral and circumorificial dermatitis, diarrhoea and alopecia (Kury et al. 2002; Ackland and Michalczyk 2006). Other putative $\mathrm{Zn}$ transporters detected in the human gut include hZip1, hZip4 and members of the SLC30A family including ZnT1 and ZnT5 (Kury et al. 2002; Cragg et al. 2005). Similar $\mathrm{Zn}$ transporters are present in the rodent gut (Jou et al. 2009).

The cellular localisation of Zip1 varies between different cells. In human adherent cells, a cytoplasmic or vesicular localisation of Zip1 (SLC39A1) has been described for PC3, HepG2 and COS-7, with partial overlap with BIP, a marker for the ER in COS-7 (Milon et al. 2001). A cytoplasmic location of Zip1 was reported in osteoclasts (Khadeer et al. 2005) and in human mesenchymal stem cells (Tang et al. 2006), while tagged hZip1 showed strong labelling in the vicinity of the plasma membrane of non-adherent K562 erythroid cells (Milon et al. 2001; Gaither and Eide 2001). The variation in 
cellular site of localisation of hZip1 raises questions about its role in directly mediating cellular $\mathrm{Zn}$ accumulation.

That the primary function of Zip1 may not be the direct acquisition of $\mathrm{Zn}$ is suggested by the expression of Zip1 in the stromal compartment of the developing mouse gut and relative lack of effect of dietary Zn on Zipl mRNA levels in the adult mouse intestine (Huang et al. 2006) and in human HT-29 colorectal cells (Gurusamy et al. 2011). Interestingly, the hZipl gene is located within the epidermal differentiation complex (Lioumi et al. 1999) and plays a key role in the differentiation of human bone cells (Khadeer et al. 2005; Tang et al. 2006). Mouse Zipl knockout studies suggest that Zip1 has an indirect function in $\mathrm{Zn}$ uptake as no adverse effects were seen although mice were unable to adapt to nutritional $\mathrm{Zn}$ deficiency (DufnerBeattie et al. 2006; Kambe et al. 2008). Thus, Zip1 may be involved in $\mathrm{Zn}$ homoeostasis through a regulatory rather than a primary role in cellular $\mathrm{Zn}$ uptake.

The purpose of this study was to establish whether hZip1 is localised to the apical plasma membrane where it could facilitate enterocyte $\mathrm{Zn}$ uptake and to employ knockdown and over expression experiments to demonstrate a role for hZip1 in cellular $\mathrm{Zn}$ uptake.

\section{Materials and methods}

\section{Human tissue}

Small intestine tissue was collected from gastroendoscopy biopsies from normal subjects. Tissue from normal resting breast was obtained from breast biopsies performed for diagnosis of breast diseases, and skin tissue was obtained from plastic surgery. The tissues were immediately frozen at $-80{ }^{\circ} \mathrm{C}$ until use. Ethical consent for this study was granted by Deakin University EC 3.2-2000 and by the Royal Children's Hospital EHR 20025 A.

\section{Cell culture}

Caco-2 human adenocarcinoma cells were cultured in $75-\mathrm{cm}^{2}$ culture flasks (Ackland et al. 2005) or on EHSmatrix (Sigma, Australia)-coated porous Transwell filters (Costar, Australia) in DMEM medium supplemented with $10 \%$ FBS (CSL, Australia). Frozen cell pellets from human colorectal adenocarcinoma HT29, duodenal adenocarcinoma HuTu80 and human brain neuroblastoma LA-1s cell lines were also used.

Treatment of cells

Cells were treated with $100 \mu \mathrm{M} \mathrm{ZnCl}, 100 \mu \mathrm{M} \mathrm{ZnCl}$ plus $0.2 \mathrm{nM}$ pyrithione, $6 \mu \mathrm{M} \mathrm{N}, \mathrm{N}, N, N$-tetrakis (2-pyridylmethyl) ethylenediamine (TPEN) (Sigma, Australia), for $48 \mathrm{~h}$ in growth media (Wilson et al. 2006). Foetal bovine serum (FBS) was incubated with analytical grade Chelex 100 resin 200-400 sodium mesh (Biorad, Australia) to remove zinc by chelation (Wilson et al. 2006). The zinc concentration in untreated and treated serum was measured by the atomic absorption spectrophotometry (Varian Spectra AA-800 (Varian, USA) and indicated a reduction in zinc content from $41 \mu \mathrm{M}$ in untreated serum to 1.2 $\mu \mathrm{M}$ in serum incubated with Chelex 100. The levels of copper and iron were unchanged with Chelex treatment. Chelex-treated serum was stored at $4{ }^{\circ} \mathrm{C}$ and added to the media when required.

hZip1 cDNA, plasmid construction and generation of transfected Caco-2 lines

The GenBank NM_014437 sequence was selected as it contained the complete open reading frame (ORF) of $h Z I P l$ gene. A small fragment of the $5^{\prime}$ region of hZIP1 ORF was amplified with primers, ZIP1-C (GGTCTGAG AGTCACTGGAGC) and ZIP1-E (GAGCGTCACGTGC AAGGCTG), from a range of cells and tissues.

The hZIPl coding sequence was amplified using the ZIP1-1F (ATAGAATTCATGGGCCTGGGGGAGAGC) and ZIP1-2R (AAATCTCGAGCTA GATTGGATGAA GAGCAG) primers containing EcoRI and XhoI restriction sites, respectively, and cloned into a pcDNA3 mammalian expression vector (Life Technologies, Australia). The pcDNA-hZIP1 plasmid was grown in and isolated from E. coli. This plasmid was transfected into Caco-2 cells using LipofectAMINE 2000 reagent (Life Technologies, Australia) following the manufacturer's instructions. The cells stably expressing the hZIP1 from CMV promoter were isolated using G418 (500 $\mu \mathrm{g} / \mathrm{ml})$ (Life Technologies, Australia) selection.

Plasmids containing a myc-tagged sequence, hZIPlmyc, were constructed through similar procedures; however, the $h Z I P l$ sequence was amplified using two primer sets to allow for insertion of the c-myc sequence into the region between transmembrane domains II and III and was predicted by TOPCONS (http://topcons.cbr.su.se/) software to face the extracellular compartment. Primers ZIP1myc1F (ATAGAATTCATGGGCCTGGGGGAGAGC) containing EcoRI site and ZIP1-myc1R (AATACTAGTC AGGTCCTCTTCAGAGATAAGTTTTTGTTCAGCCAG GTAGTCAGGCA), consisting of 20 nucleotides of complementary sequence to hZIP1 cDNA, and encoding the $c$-myc gene fragment with $B a m H I$ restriction site, were used to amplify first fragment of $h Z I P l$-myc construct. The second fragment was amplified using primers containing the BamHI site and ZIP1-myc2F (ATAACTA GTGCCATAGATGAGGCCCTGGCA) and ZIP1-myc2R 
(AAATCTCGAGCTAGATTGGATGAAGAGCAG) containing XhoI site. Both fragments were digested using EcoRI and BamHI or BamHI and XhoI restriction enzymes, respectively, and ligated together using T4 DNA ligase system (Roche, Australia) according to the manufacturer's instructions, then cloned into a pcDNA3 vector. Transfection procedures and clone selection were performed as for hZIPl construct.

siRNA: Two sets of oligonucleotides $(21-\mathrm{m})$ were designated, matching the hZIPl ORF sequence: siZIP1-1a (AAGGCTCAGCTTCCCGCCAGACCTGTCTC), siZIP11b (AATCTGGCGGGAAGCTGAGCCCCTGTCTC), siZIP1-5a (AACCCCCTCAGCCTTGCGTGCCCTGTCTC) and sizIP1-5b (AATCCTGACCCTCTCCCTGTTCCT GTCTC). Additionally, control siRNA oligonucleotides siCON-1a (AATGCATGTGTCATCGTGATACCTGTC TC) and siCON-1b (AATATGACGATGACACATGCAC CTGTCTC) lacking significant homology to any human transcript were designed. All oligonucleotides were used for in vitro transcription with T7 RNA polymerase following the manufacturer's protocol 'Silencer siRNA construction kit' (Ambion, Australia). Caco-2 cells were transiently transfected with either $50 \mathrm{nM}$ hZIPl siRNA or $50 \mathrm{nM}$ control siRNA using DMRIE-C reagent and OptiMEM media (Invitrogen Life Technologies, Australia) following the manufacturer's protocol. Cells were used for experiments $56 \mathrm{~h}$ after transfection.

\section{${ }^{65} \mathrm{Zn}$ accumulation}

The pcDNA-hZIP1, vector control and siRNA-transfected Caco-2 cell were grown to confluency in 6-well plates (Costar, Australia). Growth media were removed from cells and replaced with pre-warmed media supplemented with $1.5 \mu \mathrm{Ci} / \mathrm{ml}$ of carrier-free ${ }^{65} \mathrm{Zn}$ (Perkin Elmer, Australia), and then, cells were incubated for $60 \mathrm{~min}$. The total zinc concentration of the media was $4.1 \mu \mathrm{M}$. The cell-associated radioactivity was measured with Minaxi Auto Gamma counter, model 5530 (Packard Instrument Company, Illinois, USA). Zn accumulation was normalised to the protein concentration of lysed cells, estimated using Bio-Rad $D_{c}$ protein assay (Bio-Rad, Australia).

\section{Real-time PCR}

Real-time PCR was performed as previously described (Michalczyk et al. 2003). The primers ZIP1realA (GCCC TGAGCCTAGTAAGCTGTT) and ZIP1realB (TCATCTA TGGCAGCCAGG TAGTC) were used to amplify hZIPl fragments. Primers 18s-realA (CGGCTACCACATCCAA GGAA) and 18s-realB (GCTGGAATTACCGCGGCT) designed to the $18 \mathrm{~s}$ ribosomal sequence, an internal control, were run in parallel reactions. A DNA-free kit (Ambion, Australia) was applied to remove genomic DNA. $2^{-\Delta \Delta C T}$ and relative mRNA quantity calculations and statistical analysis ( $t$ test) were performed on all reactions.

\section{Antibodies}

The sequence encoding the $N$-terminus of hZIP1 transporter was used to raise antibodies. Briefly, the ZIP1-myc1F forward primer and GST-R reverse primer (AATCTCGAG AACTTCACCTCCAGCCCCAC) were utilised to amplify the $h Z I P l$ fragment with restriction sites for EcoRI and SpeI correspondingly. The PCR product was cloned into pGEX-2T (Amersham Pharmacia, Sweden) in frame with the carboxy terminus of the bacterial glutathione-S-transferase (GST) gene. The GST-hZIP1 fusion protein was expressed in E. coli according to the manufacturer's protocol. The GST-hZIP1 fusion protein was used to raise antibodies (hZip1-FP) to the hZIP1 transporter in sheep (Institute of Medical and Veterinary Science, Adelaide, South Australia).

The following commercially available antibodies and reagents were used: monoclonal anti-myc antibody (Roche, Melbourne, Australia), monoclonal anti-human transferrin receptor antibody and phalloidin-FITC conjugant (SigmaAldrich, Australia), monoclonal anti-PDI (protein disulphide isomerase) antibody (Stressgene, Canada), monoclonal anti-villin (Immunotech, Slovak Republic), monoclonal anti-p230 (Transduction Laboratories, USA) and monoclonal anti-E-cadherin (Chemicon, Australia).

\section{Immunofluorescence}

Adherent cultured cells were grown on 10-mm-diameter glass cover slips and processed for immunostaining as previously described (Michalczyk et al. 2003). Briefly, selected primary antibodies were diluted in $1 \%$ BSA in PBS and applied to cells overnight at $4{ }^{\circ} \mathrm{C}$. Control cells were incubated with ZIP1-FP fusion protein overnight. After 3 PBS washes, a secondary antibody AlexaFluor 488 donkey antisheep IgG or donkey anti-mouse IgG (Molecular Probes, USA), 1 in 2000 dilution was applied for $2 \mathrm{~h}$ at room temperature. Additional Cy-5 donkey anti-sheep or donkey antimouse secondary antibodies (Chemicon, Australia) were added to cells when double label was required. To label only the surface pool of hZIP1-myc, hZIP1 and transferrin receptor, the Triton $\mathrm{X}-100$ permeabilisation step was omitted and cells were immunolabelled as described above. Plasma membrane binding and internalisation of anti-hZIP1, antimyc and anti-transferrin receptor antibodies were detected when live cells were incubated with $5 \mu \mathrm{g} / \mathrm{ml}$ antibodies for $1 \mathrm{~h}$. After that, cells were fixed, permeabilised and processed as described previously. Cells were viewed using a Leica TCS SP2 AOBS laser confocal microscope (Leica, 
Australia), with PlanApo 100x oil objective at RT. Images were viewed and captured on a HP workstation with Leica Microsystems TCS SP2 software (Leica, Australia). Image overlay, brightness, contrast and colour balance adjustments were done using Photo Paint software version 6.0.

\section{Statistics}

Data are presented as mean \pm SD. Statistical analyses were carried out using a $t$ test with unequal variance.

\section{Results}

hZip1 was expressed endogenously in the human small intestine and in cultured human gut cell lines

Expression of hZipl mRNA was detected in extracts from human small intestine and in extracts from cultured gut cells. For comparison, we also investigated hZip1 in other human tissues including breast and skin, and in cultured human skin fibroblasts and lymphoblasts, using RT-PCR. A band of size 341 bp was detected in all samples (Fig. 1).

hZip1 protein had a perinuclear distribution in nondifferentiated Caco- 2 cells that was not influenced by extracellular zinc conditions

The localisation of endogenous hZip1 protein in Caco-2 cells was determined. An antigen/antibody competition

\section{0}

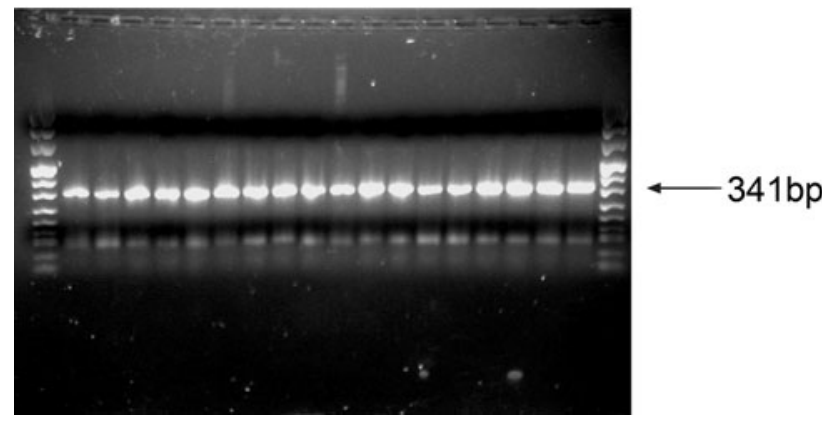

Fig. 1 hZip1 gene product was detected in a wide range of human tissues and cell lines. a A band (341 bp) representing the $5^{\prime}$ region of the hZIP1 ORF was found in all tissues and cells tested using RTPCR. PMC42 (human breast adenocarcinoma) cells (lane 2), Caco-2 (human colon adenocarcinoma) cells (lane 3), HT29 (human colon adenocarcinoma) cells (lane 4), HuTu80 (human duodenum adenocarcinoma) cells (lane 5), LA-1 s (human neuroblastoma) cells (lane 6), fibroblasts 1 (lane 7), fibroblasts 2 (lane 8), fibroblasts 3 (lane 9), fibroblasts 4 (lane 10); fibroblasts 5 (lane 11), lymphoblasts 1 (lane 12), lymphoblasts 2 (lane 13), lymphoblasts 3 (lane 14), small intestine biopsy 1 (lane 15), small intestine biopsy 2 (lane 16), resting breast tissue 1 (lane 17); resting breast tissue 2 (lane 18), finger skin tissue (lane 19) control showed no staining (Fig. 2A, a). A pattern of fine cytoplasmic granules concentrated in the perinuclear region was detected in Caco-2 cells probed with hZip antibody (Fig. 2A, b). Similar patterns of distribution of hZip1 were seen in Caco- 2 cells treated for $48 \mathrm{~h}$ with $\mathrm{Zn}$ (Fig. 2A, c) and $\mathrm{Zn}$ with pyrithione (Fig. 2A, d) or subjected to zincdeficient conditions including TPEN (Fig. 2A, e) and Chelex-treated serum (Fig. 2A, f). The XZ sections confirmed the cytoplasmic localisation of hZip1 in untreated cells (Fig. 2A, b') and cells exposed to Zn (Fig. 2A, c'), Zn with pyrithione (Fig. 2A, d'), TPEN (Fig. 2A, e') and grown in Chelex-treated media (Fig. 2A, f').

In Caco-2 cells, hZip1 mRNA decreased in response to $\mathrm{Zn}$ treatment and increased in response to $\mathrm{Zn}$ depletion

The responsiveness of $h Z i p l$ mRNA to various $\mathrm{Zn}$ treat-

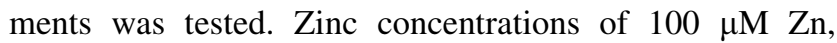
$100 \mu \mathrm{M} \mathrm{Zn}$ and $0.2 \mathrm{nM}$ pyrithione, $6 \mu \mathrm{M}$ TPEN and Chelex-100-treated serum were added to Caco-2 cells for 48 h. Additionally, Caco- 2 cells were grown on Transwell filters coated with EHS matrix to induce cell differentiation. A significant reduction in hZipl mRNA levels in response to zinc and zinc with ionophore treatment (2.7fold with $\mathrm{Zn}, 3.2$-fold with $\mathrm{Zn}$ and ionophore) was found, while zinc-deficient condition significantly increased hZipl transcript abundance (6.4-fold with TPEN, 5.8-fold with Chelex-treated serum) (Fig. 2B). Differentiated Caco-2 demonstrated a slight (2.1-fold) but significant increase in quantity of hZipl mRNA (Fig. 2B).

In non-differentiated Caco-2 cells, hZip1 was partially localised to the ER compartment

To identify the subcellular compartment in which endogenous hZip1 was localised, antibodies directed against proteins of known localisation within the cell were used together with the hZip1 antibody in Caco-2 cells (Fig. 3a, e, i, m, green). Nuclei were stained with ethidium bromide and pseudo-coloured in blue (Fig. 3c, $\mathrm{g}, \mathrm{k}, \mathrm{o})$. hZip1 protein in Caco-2 cells showed a cytoplasmic granular label (gr) with brighter perinuclear staining (pr) in some cells (Fig. 3a, e, i, m) in contrast to the junctional label seen with E-cadherin (Fig. 3b) with no colocalisation between Zip1 and E-cadherin (Fig. 3d). The transferrin receptor showed a punctate cytoplasmic pattern with some larger granules visible (lg) (Fig. 3f), and the overlay (Fig. 3h) showed no colocalisation between hZip1 and transferrin receptor labels. The immunostaining of the p230 protein (Fig. 3j), which is localised to the trans-Golgi compartment, was distinctively different from that of hZip1 

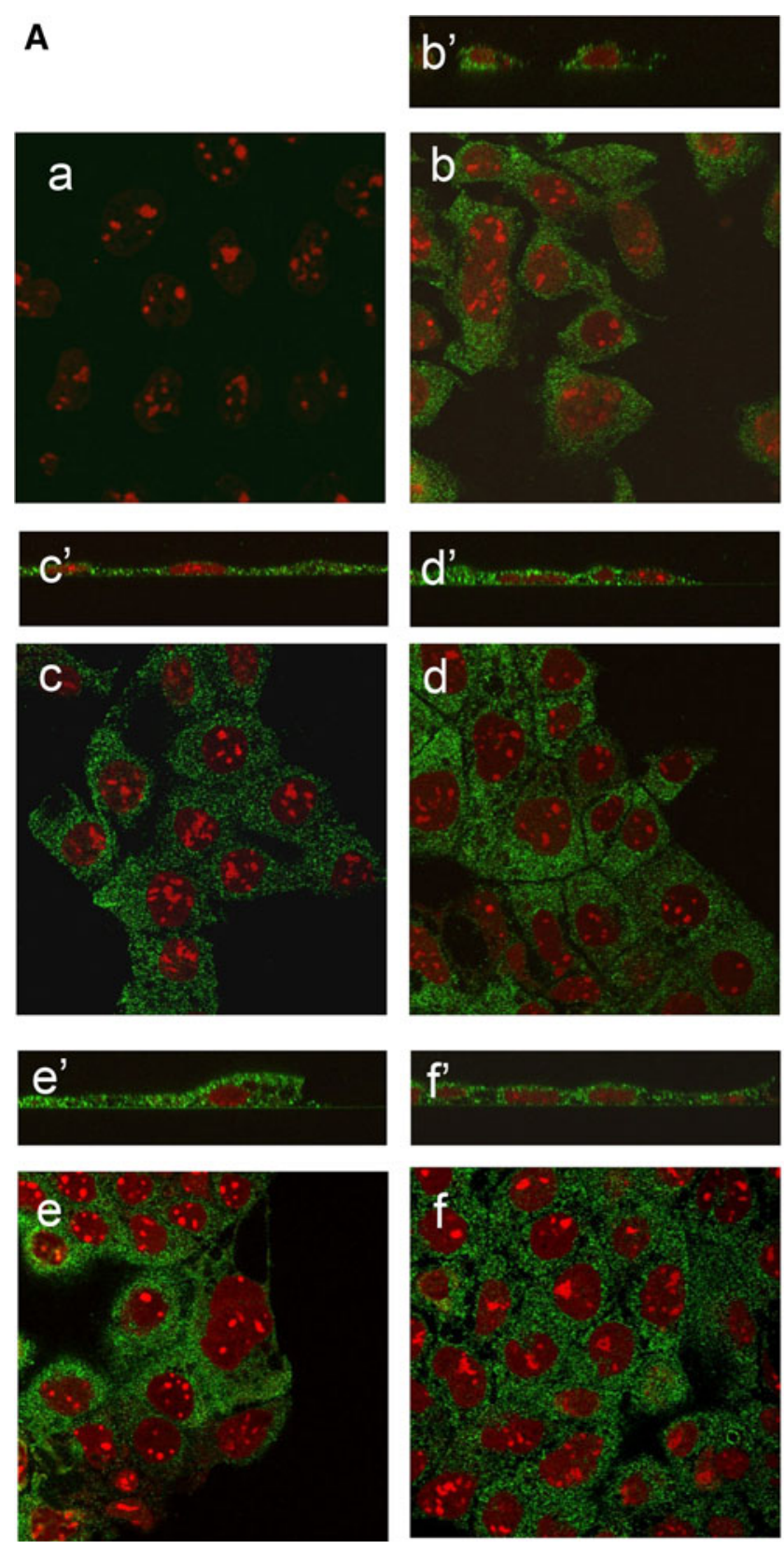

Fig. 2 Subcellular localisation of hZip1 protein in Caco-2 cells was not altered by zinc levels but differences in the expression of endogenous hZipl mRNA were detected in response to changes in zinc treatment and differentiation. A Caco-2 cells were grown in control medium (b), Zn-supplemented (c and d) or Zn-deficient (e and f) media for $48 \mathrm{~h}$ and incubated with hZip1 Ab. Cells were stained with hZip1 antibody with fourfold excess of antigen to test the Ab specificity (a). Untreated medium (b), medium supplemented with $100 \mu \mathrm{M}$ zinc (c), medium with $100 \mu \mathrm{M}$ zinc and $0.2 \mathrm{nM}$ pyrithione (d) zinc-deficient medium with $6 \mu \mathrm{M}$ TPEN (e) and also Chelex-

seen in the overlay (Fig. 31). The immunostaining of PDI (Fig. 3n), a resident protein of the ER, showed a cytoplasmic granular label (gr) with brighter perinuclear staining in some cells (pr). The overlay with

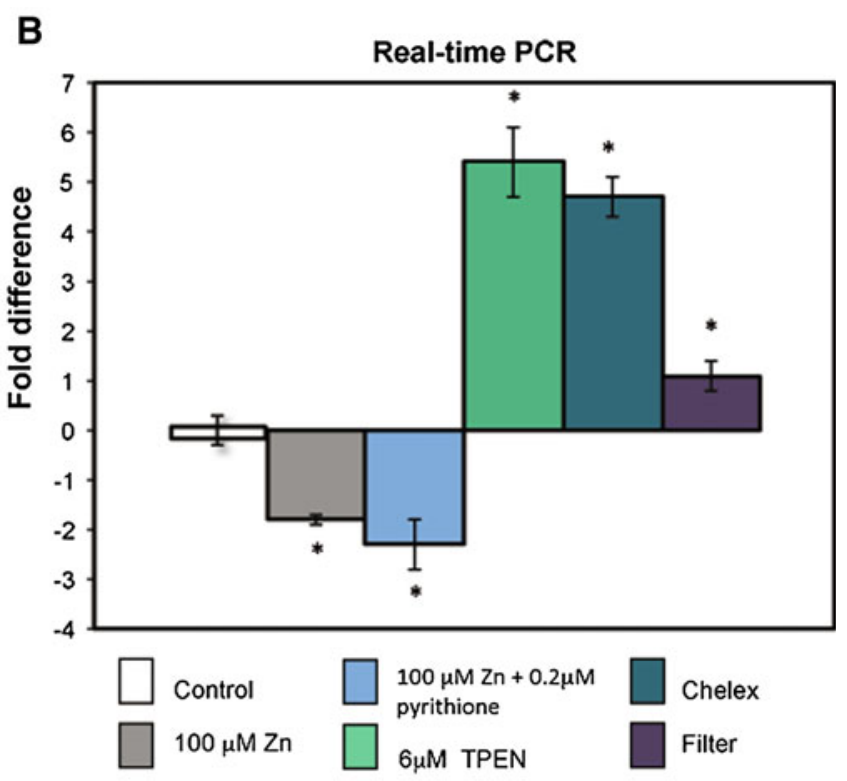

treated medium (f). The XZ sections (b'-f'). B Real-time PCR analysis showed down-regulation on hZipl mRNA in Caco-2 cells exposed to $100 \mu \mathrm{M}$ zinc (grey bar) and $100 \mu \mathrm{M}$ zinc with $0.2 \mathrm{nM}$ pyrithione (blue bar), while $6 \mu \mathrm{M}$ TPEN treatment (light green bar) and incubation with Chelex-treated media (dark green bar) resulted in increased levels of $h Z I P 1$ transcript. In differentiated Caco-2 upregulation, the hZipl levels were detected (dark grey bar) compared to the control (solid white bar). The bars represent the mean $( \pm \mathrm{SD})$ of 3 independent experiments. Stars indicate the statistically significant differences $(p<0.05, t$ test $)$

hZip1 protein (Fig. 3p) demonstrated partial colocalisation (yellow colour) between hZip1 and PDI labels, especially in perinuclear region (pr) and in granules located close to this region $(\mathrm{pg})$. 

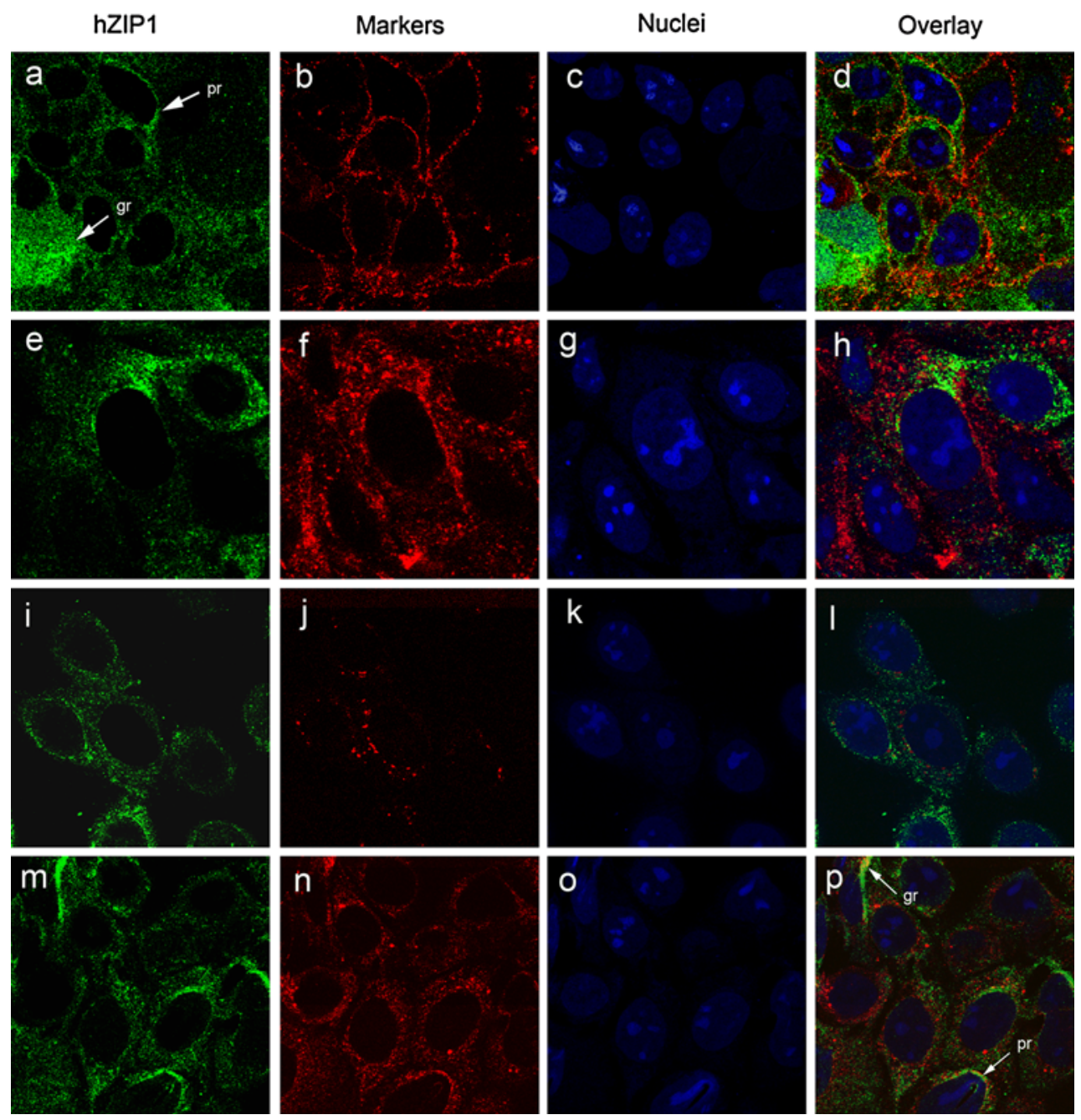

Fig. 3 hZip1 was partially colocalised to the ER in non-differentiated Caco-2 cells. hZip1 protein showed cytoplasmic granular $(g r)$ and perinuclear staining in some cells $(p r)(\mathbf{a}, \mathbf{e}, \mathbf{i}$ and $\mathbf{m})$. E-cadherin showed a punctate cell junction pattern (b) with no colocalisation between hZip1 and E-cadherin (d). Transferrin receptor was detected as a punctate cytoplasmic pattern (f). The overlay showed no colocalisation between hZip1 and transferrin receptor (h). trans-Golgi

marker p230 demonstrated perinuclear label (j) with no colocalisation with hZip1 (I). PDI protein localisation was similar to hZip1 (n) with partial colocalisation (yellow colour) between these proteins was identified in perinuclear region $(p r)$ and in granules located close to this region $(p g)$. Cell nuclei were stained with ethidium bromide (c, $\mathbf{g}$, $\mathbf{k}$ and $\mathbf{o}$ )

\section{In differentiated Caco-2 cells, hZip1 was localised}

beneath the microvilli

To investigate the localisation of hZip1 in differentiated gut epithelia that more closely resemble enterocytes, Caco- 2 cells were grown on EHS-coated Transwell filters for 3 weeks to induce differentiation and then stained with the hZip1 antibody and cellular markers. E-cadherin label was detected at the cell junctions (Fig. 4g), while hZip1 had a granular cytoplasmic distribution (Fig. 4d). There was no significant colocalisation between E-cadherin and hZip1 proteins within differentiated Caco-2 cells in XY or XZ sections (Fig. $4 \mathrm{j}, \mathrm{j}$ '). F-actin was expressed mainly at the vicinity of the apical region of the cells (Fig. 4h) and showed some overlap with

hZip1 (Fig. 4k, k'). Villin was detected only at the apical surface of Caco-2 cells marking the position of microvilli (Fig. 4i). The hZip1 label appeared to overlap with villin (Fig. 4l) but the XZ section showed that hZip1 was present beneath the microvilli (Fig. 4l'). Our hZip1 immunostaining shows that it is possible that in differentiated cells, some Zip1 protein remains within the Golgi network.

Internalisation studies indicated that hZip1-myc protein was not present at the plasma membrane of Caco-2 cells

To confirm that hZip1 was not located at the plasma membrane but adjacent to the apical surface in 
Fig. 4 hZip1 protein was not localised to the cell junction in differentiated Caco- 2 cells. Permeabilised, differentiated Caco- 2 cells were stained with hZip1 antibody and various cellular markers. Focal plane of the cells (a, b and $\mathbf{c})$. hZip1 at the junctional region between cells (d) but more dispersed than E-cadherin at cell junctions (g). Partial colocalisation between hZIP1 and E-cadherin when overlayed (j). XZ section showing proteins present in different regions (j'). hZip1 showed a diffuse, uniform pattern at the apical surface (e). F-actin was found at cell junctions (h). hZIP1 and F-actin showed no colocalisation ( $\mathbf{k}$ and k'). Villin highlighted the apical microvilli (i). In the same region, hZip1 (f) demonstrated a similar pattern (l) but the XZ section clearly identified villin in the region above hZip1 (l') a
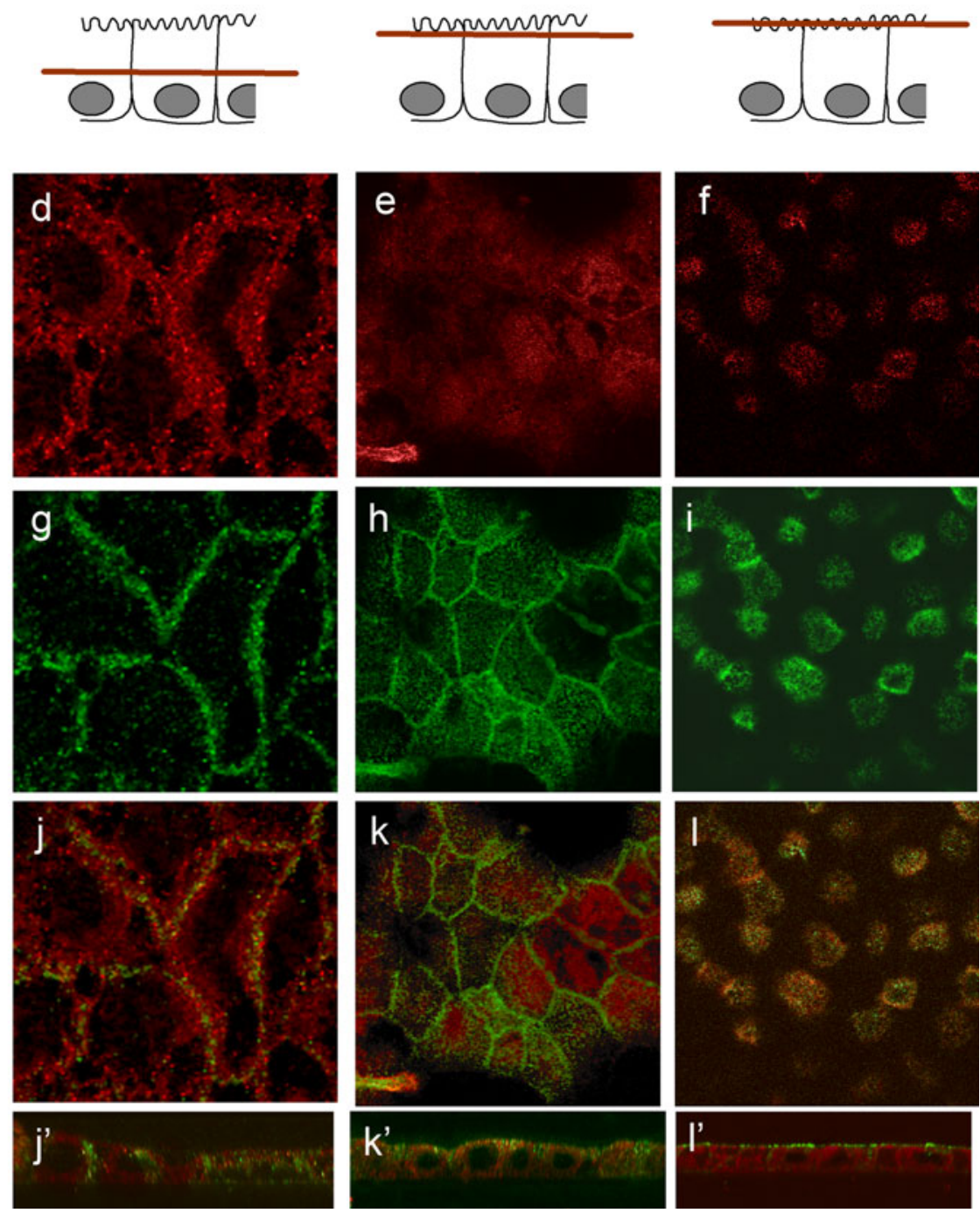

differentiated cells, a myc-tag was incorporated into the predicted extracellular loop of hZip1 protein and the construct introduced into Caco- 2 cells. Caco- 2 cells expressing the hZip1-myc were used for trafficking experiments to enable us to determine whether the hZip1 protein resided at the plasma membrane or alternatively, near the plasma membrane. Western blot analysis of extracts from Caco-2 cells transfected with pcDNA3-hZip1-myc showed a band of approximately $35 \mathrm{kDa}$, the expected size for hZIP1-myc protein, with anti-myc antibodies (Fig. 5A lane 2) but not in the vector control (Fig. 5A lane 1). In the permeabilised Caco-2 cells, hZip1-myc showed fine-granular, cytoplasmic label (Fig. 5Bc) not seen in cells transfected with vector alone (Fig. 5B, a). In Caco-2 cells, no staining was found on the surface of the non-permeabilised cells (Fig. 5B, d) proving that hZip1 was not localised to the plasma membrane.
To test the possibility that hZip1 may be located to the plasma membrane under conditions of zinc deficiency and subsequently endocytosed by cells, the Caco-2-hZip1-myc cells were incubated in either growth medium or $6 \mu \mathrm{M}$ TPENsupplemented growth medium in the presence of $5 \mu \mathrm{g} / \mathrm{ml}$ anti-myc antibodies for $60 \mathrm{~min}$ and then processed for immunofluorescence. Antibody $(5 \mu \mathrm{g} / \mathrm{ml})$ to the extracellular region of the transferrin receptor, a protein which traffics between plasma membrane and early endosomes, was used as internalisation control for the Caco-2-hZip1-myc cells. The hZip1-myc protein was not detected on the surface or internalised by Caco- 2 cells grown in either zinc-sufficient or zincdeficient media (Fig. 5C, a, b). However, the transferrin receptor showed strong granular cytoplasmic staining including plasma membrane label under these two conditions (Fig. 5C, c and $d)$. These results do not support the idea of constitutive or zinc-induced cycling of hZip1 to the plasma membrane. 

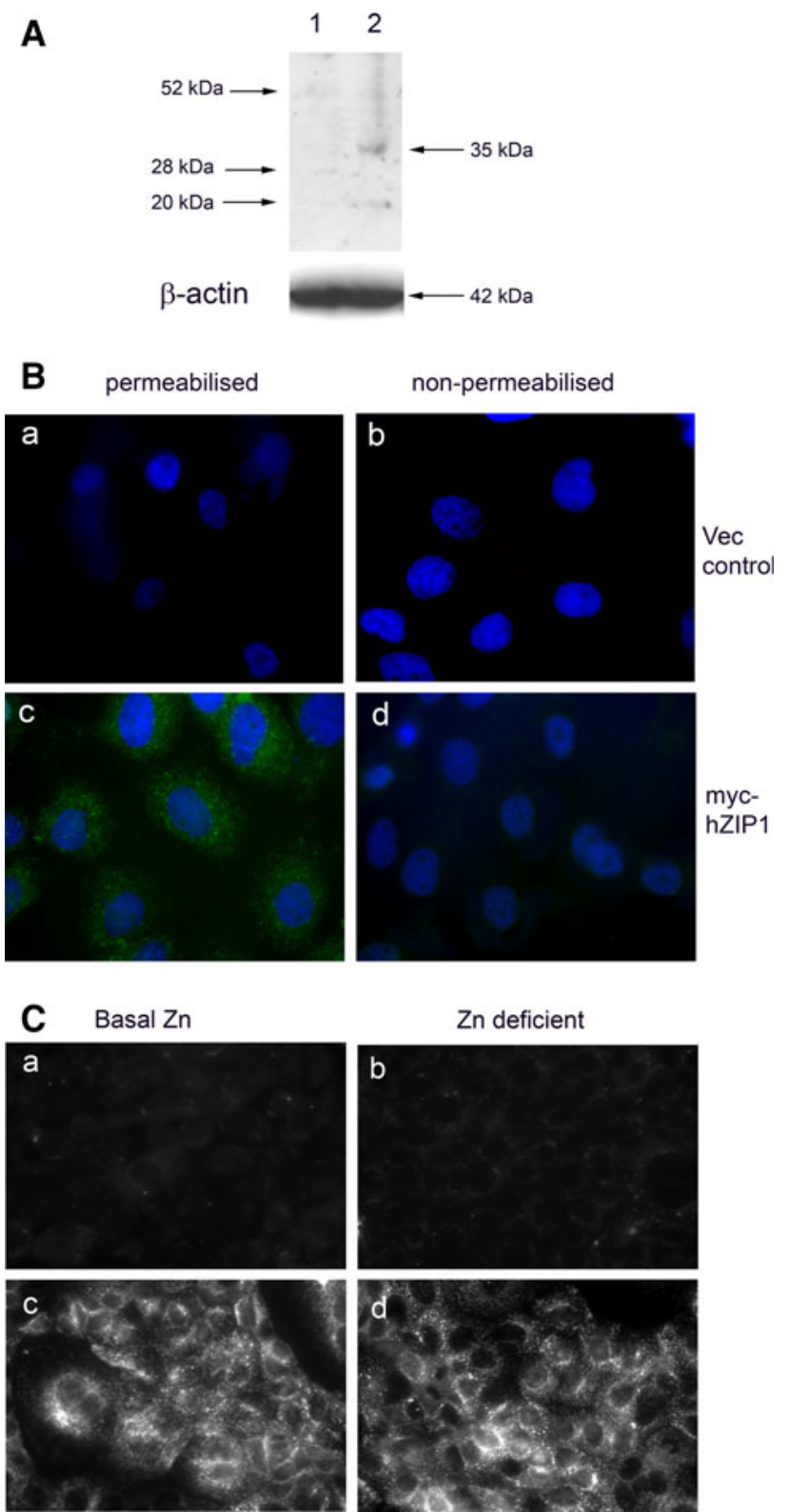

Fig. 5 The hZip1-myc protein was not detected at the plasma membrane of Caco-2 cells, and hZip1-myc-tagged protein was not internalised by the cells. A Western blot analysis of the Caco- 2 cell extracts probed with anti-myc antibody, with $\beta$-actin expression as loading control, representative of 3 independent experiments. Lane 1, Caco-2 cells transfected with pcDNA3 vector control; Lane 2, Caco-2 cells transfected with hZip1-myc construct, showing $35 \mathrm{kDa}$ band of expected size. B immunolocalisation of hZip1 protein in Caco-2 cell line. Permeabilised $(\mathbf{a}, \mathbf{c})$ and non-permeabilised $(\mathbf{b}, \mathbf{d})$ cells were labelled with anti-myc antibody. No staining in vector control Caco-2 cells (a and b). No label in non-permeabilised Caco-2 (d) cells. Permeabilised Caco-2 cells had a granular cytoplasmic pattern (c). C Live cells were incubated with $5 \mu \mathrm{g} / \mathrm{ml}$ anti-myc or anti-transferrin receptor antibodies for $1 \mathrm{~h}$. The hZip1-myc Caco-2 cells were either maintained in growth media $(\mathbf{a}, \mathbf{c})$ or in media supplemented with 6 $\mu \mathrm{M}$ TPEN (b, d). Surface binding or internalisation of anti-myc antibody was not detected in cells $(\mathbf{a}, \mathbf{b})$ while anti-transferrin receptor antibody was found at the plasma membrane and in the cytoplasm $(\mathbf{c}$,
Overexpression of hZip1 transporter increased Zn accumulation in non-differentiated Caco- 2 cells

To determine the role of hZip1 in zinc transport, the complete Zipl ORF was cloned into the pcDNA3 mammalian expression vector and stably expressed from a CMV promoter in Caco-2 cells. Real-time PCR was used to quantify hZip1 transcripts using the 18 s ribosomal subunit as an internal control. In hZipl overexpressing Caco-2 cells, the hZipl levels were significantly increased to three times that of the vector-only cells (Fig. 6a). To verify whether overexpression of hZip1 was associated with increased zinc accumulation, cells were exposed to ${ }^{65} \mathrm{Zn}$ in DMEM medium supplemented with $10 \%$ FBS for $1 \mathrm{~h}$. Accumulation of zinc in Caco-hZip1 cells $(208.5 \mathrm{pmol} \mathrm{Zn/}$ $\mathrm{mg}$ protein) was significantly increased by twice relative to that of the Caco-2-vector control cells $(115 \mathrm{pmol} \mathrm{Zn} / \mathrm{mg}$ protein) $(p<0.05, t$ test) (Fig. 6b).

Knockdown of hZip1 reduces $\mathrm{Zn}$ accumulation in non-differentiated Caco-2 cells

A function for the endogenous hZip1 protein in transporting zinc was investigated by assessing the effect of inhibition of $h Z I P l$ expression on ${ }^{65} \mathrm{Zn}$ accumulation. Caco-2 cells were transfected with two different siRNAs designed to hybridise with coding region of the hZipl mRNA and to cause degradation of the transcript by the RNA-induced silencing complex. The hZipPl mRNA level, estimated by real-time PCR, was significantly reduced by 2.2 -fold when the cells were treated with $50 \mathrm{nM}$ of siRNA hZipl compared to cells treated with $50 \mathrm{nM}$ randomly selected control siRNAs, with no similarity to any known human mRNA (Fig. 6c). The ${ }^{65} \mathrm{Zn}$ accumulation in hZipl siRNA-transfected Caco- 2 cells was $20 \%$ lower than in control siRNA cells $(p>0.05, t$ test) (Fig. 6d).

\section{Discussion}

Zip1 is one of the 14 members of the SLC39 putative Zn transporters that are postulated, but not conclusively shown to mediate cellular accumulation of $\mathrm{Zn}$. We detected hZipl mRNA transcripts in human intestinal tissue and in a cultured human gut line as well as in other human tissues including breast and skin and cultured human skin fibroblasts and lymphoblasts, indicating widespread expression of the human gene that is consistent with the expression in mouse tissues (Dufner-Beattie et al. 2003). An explanation for the presence of multiple members of the SLC39A is not clear but it would seem that the role of these transporters may extend beyond that of a transmembrane $\mathrm{Zn}$ transporter. 
Fig. 6 Zinc accumulation was increased in Caco-2 cells overexpressing the hZip1 construct and decreased in cells transfected with hZipl siRNA. a hZipl mRNA expression levels in Caco-2 cells transfected with hZip1 constructs were higher relative to vector. b Zinc accumulation was increased in Caco-2 cells transfected with hZipl construct relative to vector-only following incubation in ${ }^{65} \mathrm{Zn}$. c hZipl mRNA expression levels in Caco-2 cells transfected with hZipl siRNA was reduced relative to control siRNA. d Zinc accumulation in Caco-2 cells transfected with hZipl siRNA was reduced compared to control siRNA following incubation in ${ }^{65} \mathrm{Zn}$. The bars represent the mean $( \pm \mathrm{SD})$ of 3 independent experiments. Stars indicate the statistically significant difference $(p<0.05$ $t$ test)

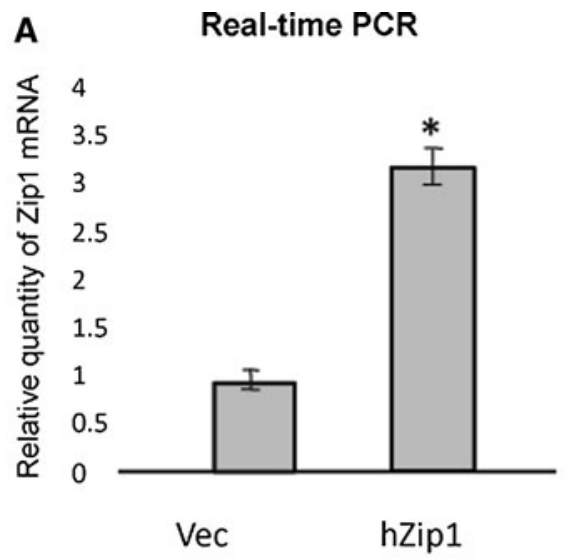

B

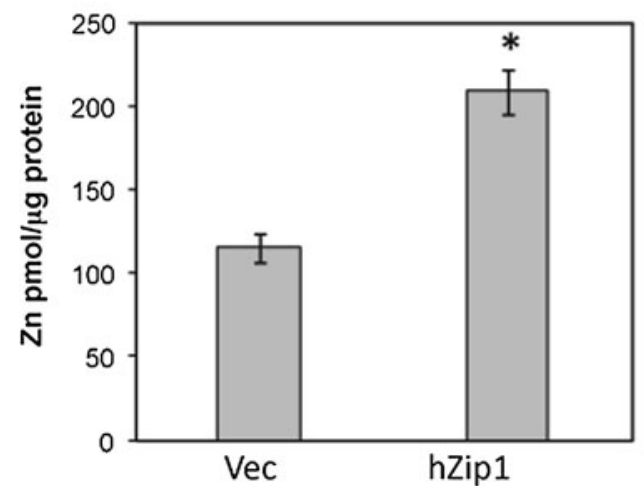

C

Real-time PCR

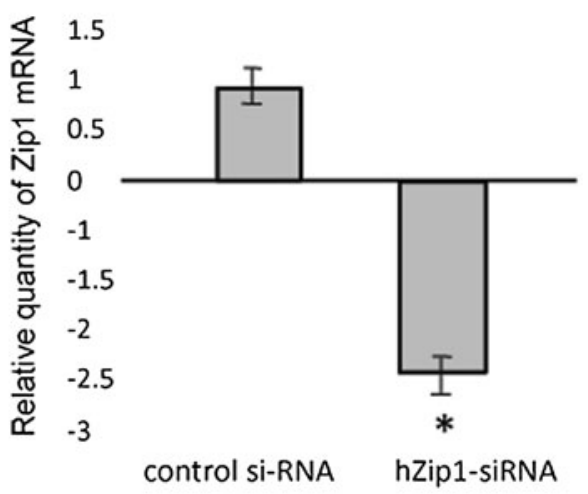

D

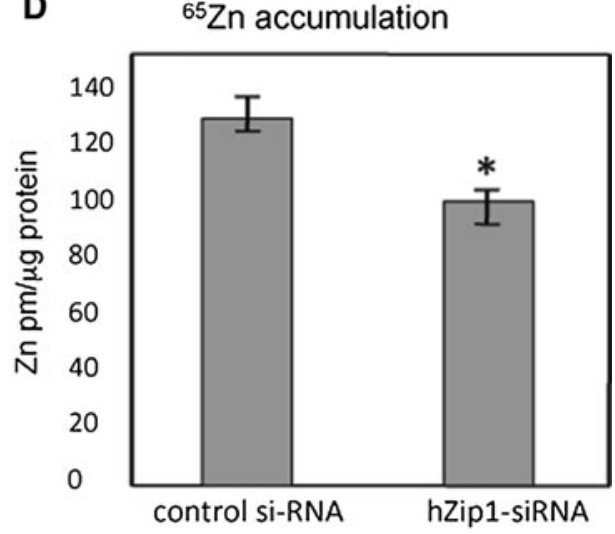

In cultured human Caco-2 cells, endogenous hZip1 protein had a cytoplasmic, vesicular location that partially overlapped with the endoplasmic reticulum marker PDI, similar to the localisation seen in other studies (Milon et al. 2001; Khadeer et al. 2005). In contrast, in Caco-2 cells that were differentiated by growth on EHS matrix-coated filters, the Zip1 protein was detected in the vicinity of the apical membrane. This indicates that the change in Zip-1 localisation was associated with the differentiated state. It is consistent with previous studies showing that the extracellular matrix is essential for proper differentiation (Schock and Perrimon 2002), and the induction of genes is required for the formation of differentiated enterocytes (Halbleib et al. 2007; Saaf et al. 2007) and for targeting of molecules to the appropriate domain in enterocytes (Schreider et al. 2002). Previous studies with Caco-2 cells have shown that hZip1 expression is influenced by the differentiation state of the cells (Zemann et al. 2010). Our data have parallels with previous findings on the role of frataxin in iron transport in differentiated Caco- 2 cells. The authors reported that the frataxin localisation changed during Caco-2 cell differentiation, which was attributed to its role in iron intestinal uptake in differentiated cells. It was concluded that differentiated enterocytes are specialised for absorption and transport of iron and differ from the undifferentiated precursors in their expression of iron uptake and iron transport proteins (Acquaviva et al. 2005). The change in localisation of hZip1 in Caco-2 cells could be linked to zinc fluxes, in relation to changes in enterocyte metal absorption following their differentiation.

Colocalisation studies of differentiated Caco-2 cells indicated that although hZip1 was apically located, it did not reside at the plasma membrane but was beneath the microvilli marker, villin. Our functional data indicate no association of the hZip1 protein with the apical plasma membrane. This was apparent from experiments in Caco-2 cells transfected with a myc epitope predicted to be placed in extracellular region (TOPCONS topology software) that showed no hZip1 staining in non-permeabilised or live cells that had been incubated with an anti-myc antibody. Furthermore, endogenously expressed hZip1 protein was not detected at the cell surface with the antibody to the extracellular N-terminus of hZip1 protein, even if the cells were grown in a zinc-deficient environment. In contrast, under the same experimental conditions, the transferrin receptor was detected on the cell surface from where it was internalised into cytoplasmic vesicles.

We found that the localisation of the hZip1 protein was not altered in response to changes in extracellular zinc levels. This is consistent with a similar study on Caco-2 
cells (Shen et al. 2008) but in contrast to the intracellular trafficking of hZip1 protein reported in transfected human embryonic kidney cells (Wang et al. 2004) and in CHO cells (Huang and Kirschke 2007). Thus, hZip1 Zn responsiveness appears to be cell type specific.

Although we did not detect the hZip1 protein at the plasma membrane of differentiated Caco- 2 cells, where it would be predicted to be if it was involved in $\mathrm{Zn}$ uptake, we demonstrated a $\mathrm{Zn}$ transport role for the endogenous protein by the $20 \%$ reduction in zinc accumulation in hZipl knockdown cells. When hZipl was overexpressed in Caco- 2 cells, ${ }^{65} \mathrm{Zn}$ accumulation was increased by twofold. This is similar to other studies in human K562 erythroleukemia cells (Gaither and Eide 2001) and PC-3 prostate cells (Franklin et al. 2003) where overexpression of Zipl induced $\mathrm{Zn}$ accumulation and the rate of $\mathrm{Zn}$ uptake. Thus, our study as well as those of others unequivocally demonstrates that Zip1 plays a role in $\mathrm{Zn}$ accumulation.

We tested the $\mathrm{Zn}$ responsiveness of hZipl in differentiated Caco- 2 cells by exposing them to excess $\mathrm{Zn}(100 \mu \mathrm{M}$ $\mathrm{Zn}$ and $100 \mu \mathrm{M} \mathrm{Zn}+$ zinc ionophore) and to $\mathrm{Zn}$ depletion (Zn chelator and Chelex-100-treated serum). Significant decreases in hZipl mRNA were seen in response to excess $\mathrm{Zn}$, while $\mathrm{Zn}$ deficiency induced increase in the levels of hZipl mRNA. It should be noted that in the mouse, intestine dietary levels of $\mathrm{Zn}$ did not affect the abundance Zip1 mRNA or protein (Huang et al. 2006; Dufner-Beattie et al. 2003), contrary to the human gut, where the response to dietary $\mathrm{Zn}$ differs from the rodent model (Cragg et al. 2005).

To account for our findings and explain the function of hZip1 in human enterocytes, we developed a hypothetical model. $\mathrm{Zn}$ is taken up by ion channels such as the $\mathrm{K}^{+} / \mathrm{Zn}^{2+}$ antiporter (Ackland and McArdle 1990,1996) or the $\mathrm{Na}^{+} /$ $\mathrm{Zn}^{2+}$ counter transporter (Ohana et al. 2004) or transporters such as Zip4 (Wang et al. 2002) and the multi-ion importer DCT1/DMT1/Nramp2 (Gunshin et al. 1997); however, the affinity of DMT1 for zinc is low (Yamaji et al. 2001). Imported zinc is delivered to zinc-dependant proteins and sequestered into vesicles by SLC30/CDF transporters including ZnT2 (Palmiter et al. 1996a), ZnT3 (Palmiter et al. 1996b) and ZnT4 (Michalczyk et al. 2002; Murgia et al. 1999). The zinc-containing vesicles or zincosomes may be utilised for temporary metal storage or directed into an exocytotic pathway. Zinc is released from vesicular storage by SLC39A transporters in the vesicular membrane in response to decreases in cytoplasmic $\mathrm{Zn}$ levels.

In the cytoplasmic loop between transmembrane domains III and IV, two histidines conserved within the SLC39A family are potential Zn-binding regions required for $\mathrm{Zn}$ transport (Milon et al. 2006) and may act as a zinc sensor (Fig. 7) similar to the zinc-binding region in the yeast ZAP1 transcription regulator (Bird et al. 2000). If intracellular levels of $\mathrm{Zn}$ increase, more $\mathrm{Zn}$ is bound by hZip1 and this prevents release of stored vesicular $\mathrm{Zn}$ into the cytoplasm. It is also possible that hZip1, activated by zinc binding, induces additional mechanisms controlling the vesicular zinc storage, therefore contributing to intracellular zinc homoeostasis. When the cytoplasmic Zn levels fall, less $\mathrm{Zn}$ is bound to hZip1, resulting in a translocation of the metal from vesicles to the cytoplasm (Fig. 7). The feasibility of this hypothetical model is indicated by examples of similar systems involving $\mathrm{Zn}$ sensors, including the store-operated calcium channel (SOCC) where Zn inhibits calcium influx (Gore et al. 2004) and Yiip, a member of the SLC30 family which effluxes Zn in response to the activation of the cytoplasmic domain ( $\mathrm{Lu}$ and Fu 2007; Nies 2007).

Based on our experimental data, we propose a hypothesis that would explain the observations of higher intracellular $\mathrm{Zn}$ concentrations in cells overexpressing hZip1 compared to control cells. In hZip1 overexpressing cells, there is an increased number of hZip1 molecules present on the vesicular membranes that would bind zinc by the
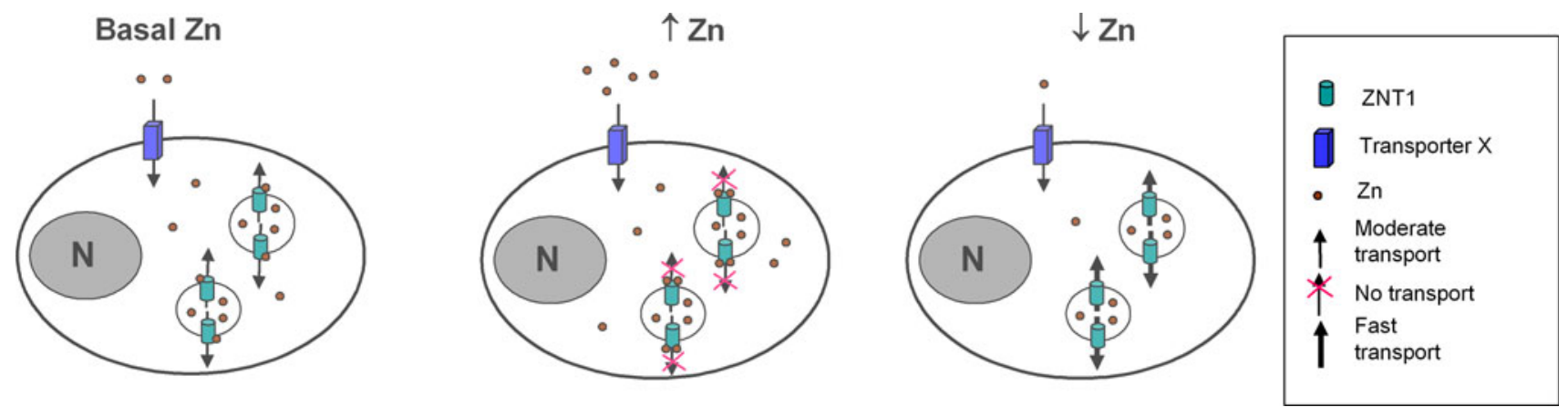

Fig. 7 A model for hZIP1 as intracellular zinc sensor in Caco-2 cells. a Zinc is taken up from the extracellular environment by transporter $\mathrm{X}$. To maintain intracellular zinc homoeostasis, endogenous hZIP1 regulates zinc release from zinc-containing vacuoles (zicosomes) into the cytoplasm in basal zinc and zinc-deficient conditions by utilising 2 cytoplasmic histidines as zinc concentration sensors. This transport is ceased following an increase in extracellular/cytoplasmic zinc levels as the hZIP1 sensors are saturated 
histidine/sensor region. The $\mathrm{Zn}$ in the cytoplasm binds to hZip-1 until saturation of histidines on additional exogenous hZip1 is achieved and $\mathrm{Zn}$ efflux from the vesicles is prevented. In addition, hZip1 may also induce activation of additional mechanisms to facilitate zinc transport into vesicles that could account for the overall increase in zinc accumulation in the hZip1 overexpressing cells. The silencing of the hZipl gene produces the opposite effect, where fewer transporters are available to bind $\mathrm{Zn}$; therefore, equilibrium is established at a lower $\mathrm{Zn}$ concentration. As a regulator of zinc fluxes, hZip1 may also contribute to zinc signalling within the cells in addition to its role in calcium regulation (Maret 2001, 2011; Hershfinkel et al. 2001).

In conclusion, the hZip1 protein was not a resident of the apical plasma membrane and does not directly facilitate $\mathrm{Zn}$ accumulation in differentiated Caco- 2 cells, although it is zinc-responsive. It is possible that hZip1 may function as a $\mathrm{Zn}$ sensor that has a regulatory role in zinc homoeostasis in human gut enterocytes, similar to that of SOCC or Yiip.

Acknowledgments This work was supported by Australian Research Council Project Grant to MLA. MLA participated in experimental design, ${ }^{65} \mathrm{Zn}$ experiments, analysis of data and preparation of the manuscript. AAM contributed to experimental design, carried out the majority of the experiments and assisted with the manuscript preparation.

\section{References}

Ackland ML, McArdle HJ (1990) Significance of extracellular zincbinding ligands in the uptake of zinc by human fibroblasts. J Cell Physiol 145(3):409-413

Ackland ML, McArdle HJ (1996) Cation-dependent uptake of zinc in human fibroblasts. Biometals 9:29-37

Ackland M, Michalczyk A (2006) Zinc deficiency and its inherited disorders: a review. Genes Nutr 1:41-50

Ackland ML, van de Waarsenburg S, Jones R (2005) Synergistic antiproliferative action of the flavonols quercetin and kaempferol in cultured human cancer cell lines. In Vivo 19(1):69-76

Acquaviva F, De Biase I, Nezi L, Ruggiero G, Tatangelo F, Pisano C, Monticelli A, Garbi C, Acquaviva AM, Cocozza S (2005) Extramitochondrial localisation of frataxin and its association with IscU1 during enterocyte-like differentiation of the human colon adenocarcinoma cell line Caco-2. J Cell Sci 118(Pt 17):3917-3924

Bird AJ, Zhao H, Luo H, Jensen LT, Srinivasan C, Evans-Galea M, Winge DR, Eide DJ (2000) A dual role for zinc fingers in both DNA binding and zinc sensing by the Zap1 transcriptional activator. EMBO J 19(14):3704-3713

Cragg RA, Phillips SR, Piper JM, Varma JS, Campbell FC, Mathers JC, Ford D (2005) Homeostatic regulation of zinc transporters in the human small intestine by dietary zinc supplementation. Gut 54(4):469-478

Dufner-Beattie J, Langmade SJ, Wang F, Eide D, Andrews GK (2003) Structure, function, and regulation of a subfamily of mouse zinc transporter genes. J Biol Chem 278(50):5014250150

Dufner-Beattie J, Huang ZL, Geiser J, Xu W, Andrews GK (2006) Mouse ZIP1 and ZIP3 genes together are essential for adaptation to dietary zinc deficiency during pregnancy. Genesis 44(5): 239-251

Franklin RB, Ma J, Zou J, Guan Z, Kukoyi BI, Feng P, Costello LC (2003) Human ZIP1 is a major zinc uptake transporter for the accumulation of zinc in prostate cells. J Inorg Biochem 96(2-3): 435-442

Gaither LA, Eide DJ (2001) The human ZIP1 transporter mediates zinc uptake in human K562 erythroleukemia cells. J Biol Chem 276(25):22258-22264

Gore A, Moran A, Hershfinkel M, Sekler I (2004) Inhibitory mechanism of store-operated $\mathrm{Ca} 2+$ channels by zinc. J Biol Chem 279(12):11106-11111

Gunshin H, Mackenzie B, Berger UV, Gunshin Y, Romero MF, Boron WF, Nussberger S, Gollan JL, Hediger MA (1997) Cloning and characterization of a mammalian proton-coupled metal-ion transporter. Nature 388(6641):482-488

Gurusamy KS, Farooqui N, Loizidou M, Dijk S, Taanman JW, Whiting S, Farquharson MJ, Fuller BJ, Davidson BR (2011) Influence of zinc and zinc chelator on HT-29 colorectal cell line. Biometals 24(1):143-151

Halbleib JM, Saaf AM, Brown PO, Nelson WJ (2007) Transcriptional modulation of genes encoding structural characteristics of differentiating enterocytes during development of a polarized epithelium in vitro. Mol Biol Cell 18(11):4261-4278

Hershfinkel M, Moran A, Grossman N, Sekler I (2001) A zinc-sensing receptor triggers the release of intracellular $\mathrm{Ca} 2+$ and regulates ion transport. Proc Natl Acad Sci USA 98(20):11749-11754

Huang L, Kirschke CP (2007) A di-leucine sorting signal in ZIP1 (SLC39A1) mediates endocytosis of the protein. FEBS J 274(15):3986-3997

Huang ZL, Dufner-Beattie J, Andrews GK (2006) Expression and regulation of SLC39A family zinc transporters in the developing mouse intestine. Dev Biol 295(2):571-579

Jou MY, Hall AG, Philipps AF, Kelleher SL, Lonnerdal B (2009) Tissue-specific alterations in zinc transporter expression in intestine and liver reflect a threshold for homeostatic compensation during dietary zinc deficiency in weanling rats. J Nutr 139(5):835-841

Kambe T, Geiser J, Lahner B, Salt DE, Andrews GK (2008) Slc39a1 to 3 (subfamily II) Zip genes in mice have unique cell-specific functions during adaptation to zinc deficiency. Am J Physiol Regul Integr Comp Physiol 294(5):R1474-R1481

Khadeer MA, Sahu SN, Bai G, Abdulla S, Gupta A (2005) Expression of the zinc transporter ZIP1 in osteoclasts. Bone 37(3):296-304

Kury S, Dreno B, Bezieau S, Giraudet S, Kharfi M, Kamoun R, Moisan J-P (2002) Identification of SLC39A4, a gene involved in acrodermatitis enteropathica. Nat Genet 31(3):239-240

Lioumi M, Ferguson CA, Sharpe PT, Freeman T, Marenholz I, Mischke D, Heizmann C, Ragoussis J (1999) Isolation and characterization of human and mouse ZIRTL, a member of the IRT1 family of transporters, mapping within the epidermal differentiation complex. Genomics 62(2):272-280

Lu M, Fu D (2007) Structure of the zinc transporter YiiP. Science 317(5845): $1746-1748$

Maret W (2001) Crosstalk of the group IIa and IIb metals calcium and zinc in cellular signaling. Proc Natl Acad Sci USA 98(22): $12325-12327$

Maret W (2011) Metals on the move: zinc ions in cellular regulation and in the coordination dynamics of zinc proteins. Biometals 24:411-418

Michalczyk A, Allen JG, Blomeley RC, Ackland ML (2002) Constitutive expression of hZnT4 zinc transporter in human breast epithelial cells. Biochem J 364(Pt 1):105-113

Michalczyk A, Varigos G, Catto Smith A, Blomeley R, Ackland L (2003) Analysis of zinc transporter, hZnT4 (Slc30A4) gene expression in a mammary gland disorder leading to reduced zinc secretion into milk. Hum Genet 113:202-210 
Milon B, Dhermy D, Pountney D, Bourgeois M, Beaumont C (2001) Differential subcellular localization of hZip1 in adherent and non-adherent cells. FEBS Lett 507(3):241-246

Milon B, Wu Q, Zou J, Costello LC, Franklin RB (2006) Histidine residues in the region between transmembrane domains III and IV of hZip1 are required for zinc transport across the plasma membrane in PC-3 cells. Biochim Biophys Acta 1758(10):1696-1701

Murgia C, Vespignani I, Cerase J, Nobili F, Perozzi G (1999) Cloning, expression, and vesicular localization of zinc transporter Dri 27/ZnT4 in intestinal tissue and cells. Am J Physiol 277(6 Pt 1):G1231-G1239

Nies DH (2007) Biochemistry. How cells control zinc homeostasis. Science 317(5845):1695-1696

Ohana E, Segal D, Palty R, Ton-That D, Moran A, Sensi SL, Weiss JH, Hershfinkel M, Sekler I (2004) A sodium zinc exchange mechanism is mediating extrusion of zinc in mammalian cells. J Biol Chem 279(6):4278-4284

Palmiter RD, Cole TB, Findley SD (1996a) ZnT-2, a mammalian protein that confers resistance to zinc by facilitating vesicular sequestration. EMBO J 15(8):1784-1791 issn: 0261-4189

Palmiter RD, Cole TB, Quaife CJ, Findley SD (1996b) ZnT-3, a putative transporter of zinc into synaptic vesicles. Proc Natl Acad Sci USA 93(25):14934-14939

Saaf AM, Halbleib JM, Chen X, Yuen ST, Leung SY, Nelson WJ, Brown PO (2007) Parallels between global transcriptional programs of polarizing Caco-2 intestinal epithelial cells in vitro and gene expression programs in normal colon and colon cancer. Mol Biol Cell 18(11):4245-4260

Schock F, Perrimon N (2002) Molecular mechanisms of epithelial morphogenesis. Annu Rev Cell Dev Biol 18:463-493
Schreider C, Peignon G, Thenet S, Chambaz J, Pincon-Raymond M (2002) Integrin-mediated functional polarization of Caco-2 cells through E-cadherin-actin complexes. J Cell Sci 115(Pt 3): 543-552

Shen H, Qin H, Guo J (2008) Cooperation of metallothionein and zinc transporters for regulating zinc homeostasis in human intestinal Caco-2 cells. Nutr Res 28(6):406-413

Tang Z, Sahu SN, Khadeer MA, Bai G, Franklin RB, Gupta A (2006) Overexpression of the ZIP1 zinc transporter induces an osteogenic phenotype in mesenchymal stem cells. Bone 38(2): 181-198

Wang K, Zhou B, Kuo Y-M, Zemansky J, Gitschier J (2002) A novel member of a zinc transporter family is defective in acrodermatitis enteropathica. Am J Hum Genet 71(1):66-73

Wang F, Dufner-Beattie J, Kim BE, Petris MJ, Andrews G, Eide DJ (2004) Zinc-stimulated endocytosis controls activity of the mouse ZIP1 and ZIP3 zinc uptake transporters. J Biol Chem 279(23):24631-24639

Wilson D, Varigos G, Ackland ML (2006) Apoptosis may underlie the pathology of zinc-deficient skin. Immunol Cell Biol 84(1): $28-37$

Yamaji S, Tennant J, Tandy S, Williams M, Singh Srai SK, Sharp P (2001) Zinc regulates the function and expression of the iron transporters DMT1 and IREG1 in human intestinal Caco-2 cell. FEBS Lett 507(2):137-141

Zemann N, Zemann A, Klein P, Elmadfa I, Huettinger M (2010) Differentiation- and polarization-dependent zinc tolerance in Caco-2 cells. Eur J Nutr 50(5):379-386 